\title{
L'INTRODUCTION DE LA GESTION AXÉE SUR LE RÉSULTAT (GAR) EN MATIÈRE BUDGÉTAIRE AU SÉNÉGAL: LES CADRES DE DÉPENSES À MOYEN TERME (CDMT)
}

\author{
Par Mamour DRAME 1
}

\section{RESUME}

La démarche sénégalaise de performance budgétaire est conçue non seulement comme devant conduire à une meilleure allocation des moyens budgétaires mais également comme instituant un dispositif de pilotage ayant pour but d'améliorer l'efficacité de la dépense publique en orientant la gestion publique vers des résultats prédéfinis. Les CDMT ont été introduits au Sénégal dans le souci, d'une part de mettre à la disposition du pays, un outil permettant de mieux gérer le déficit budgétaire à travers des projections réalistes concernant les recettes et, d'autre part, de faciliter l'identification des objets de dépenses prioritaires. Leur adoption dans la gestion budgétaire sénégalaise qui repose essentiellement sur la recherche d'une intégration des politiques budgétaires et sur des objectifs basés sur la performance, est consolidée par une dynamique normative nouvelle émergente dans le droit budgétaire sénégalais et communautaire. Toutefois, le Sénégal est confronté, dans la mise en œuvre des CDMT à des contraintes à la fois d'ordre technique, structurel et humain. Ces atteintes se traduisent en pratique par un décalage entre les dotations financières inscrites dans les CDMT et celles prévues par la loi de finances. Ainsi, le Sénégal doit généralement faire des efforts dans deux sens : le cadrage macroéconomique et la programmation sectorielle. De façon plus générale, c'est une exigence démocratique impliquant un changement de culture et une modification sensible des comportements.

\section{INRODUCTION}

L'Etat présentait de manière classique ses finances sous la forme de moyens mis à la disposition de ses divers services ministériels, sans faire apparaitre la finalité de son action. L'idée de prendre en compte les buts est née en 1973, à l'instar du modèle des Etats unis, et s'est concrétisée par la création du budget fonctionnel ${ }^{2}$. Le budget fonctionnel présentait les dépenses publiques non plus par crédits affectés aux ministères, mais par grandes fonctions

1 Doctorant en Droit public, Université Gaston Berger.

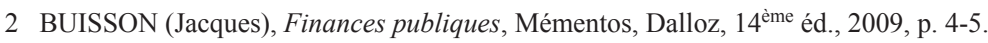


exercées par l'Etat ${ }^{3}$. Ce budget fonctionnel, séduisant par sa présentation, mais se révélant peu utile et peu efficace ${ }^{4}$ fut supprimé.

Cependant, l'idée de présenter les fonctions de l'Etat et d'en retracer l'utilité et l'efficacité en terme financier est une idée vivace et persistante, si bien qu'à la même époque sont apparus les budgets de programmes (BP), fondés sur la technique de la rationalisation des choix budgétaires $(\mathrm{RCB})^{5}$. La RCB entendait étudier les choix budgétaires de manière rationnelle et scientifique en utilisant une méthode se déroulant en trois étapes : il s'agissait d'abord, de définir les objectifs poursuivis par une action publique, de recenser ensuite les coûts et les avantages respectifs des moyens utilisés pour réaliser cet objectif, et enfin, d'en mesurer les résultats obtenus au moyen d'indicateurs systématiques. Cette technique intellectuellement attractive, a été un échec en raison des réticences d'une partie de l'administration à la mettre en œuvre et des réticences des politiques à son égard ${ }^{6}$. Aussi bien, les budgets de programme qui présentaient les dépenses publiques selon cette technique n'étaient que de simples documents d'informations des parlementaires, au demeurant peu lisibles. Apparus en France en 1970, les BP ont disparu en 1995.

Héritier de cette tradition française et soumis à des contraintes budgétaires au niveau communautaire, le Sénégal a expérimenté diverses techniques de présentation budgétaire. Sur fond de lutte contre la pauvreté, la recherche de l'efficacité constitue la ligne directrice de toutes les réformes budgétaires. En effet, dans le courant des années 1980, au Sénégal comme dans beaucoup de pays africains, le processus budgétaire est grandement affaibli par l'écart croissant entre les «besoins» et les «ressources disponibles». Les ministères opérationnels fondent leurs demandes budgétaires sur des besoins élevés, héritent ou se créent des engagements qu'ils n'ont pas les moyens de financer et doivent en même temps gérer en permanence des problèmes quotidiens créés par l'insuffisance et l'indisponibilité immédiate de crédits. Les ministères des finances sont au contraire obligés de tenir un message de rigueur et, au mieux, de reconduire les budgets précédents en l'état, préoccupés par la faiblesse des recettes intérieures, par le poids de la dette et par les déficits budgétaires. La croissance démographique, les coûts de fonctionnement des nouveaux projets, les retards de financements extérieurs et les chocs exogènes, accentuent la pression sur des budgets qui n'ont pas été correctement élaborés. De plus, tandis qu'aucun service ne veut être «victime » de compression budgétaire, il n'est fait nulle part mention de problèmes d'efficacité dans l'utilisation des fonds disponibles: les performances des services sont surtout jugées sur les intrants, sur le respect des règles, sur la manière dont les budgets alloués sont exécutés ${ }^{7}$.

3 Des fonctions comme éducation et agriculture, logement, extérieur, défense, etc.

4 Il s'agissait d'un simple document d'information remis aux parlementaires.

5 Expérimentée en France et qui s'inspirait de la technique américaine du PPBS (planning, Programming, Budgeting, system), mise en œuvre à partir de 1921.

6 La technocratie est privilégiée par rapport à la démocratie.

7 Rapport de la Banque mondiale, «Intégrer les politiques, les plans et les budgets dans un cadre à moyen terme », Chapitre 3 du manuel sur la Gestion des dépenses publiques 1998. Voire aussi P. Le 
Pour améliorer cette situation, une solution consiste à dépasser le cadre restreint d'un cycle budgétaire annuel et d'introduire une forme de gestion axée sur les résultats (GAR). Ces deux éléments sont, entre autres, au cœur de ce que l'on appelle le Cadre de Dépense à Moyen Terme $(\mathrm{CDMT})^{8}$. Ainsi, à partir des années 1990, de nombreux pays africains ont commencé à mettre en place les CDMT et le mouvement s'est ensuite amplifié au fur et à mesure que les pays élaboraient et mettaient en œuvre des Documents Stratégiques de Réduction de la Pauvreté (DSRP) dans lesquels le CDMT est programmé comme une innovation majeure dans le cadre des réformes budgétaires ${ }^{9}$. Le passage des Programmes d'Ajustements Structurels (PAS) aux Documents Stratégiques de Réduction de la Pauvreté DSRP implique un changement de perspectives ${ }^{10}$ qui trouve dans les CDMT un véhicule idéal pour mettre en cohérence Documents Stratégiques de Réduction de la Pauvreté (DSRP) et dépenses publiques ${ }^{11}$.

Cependant, au bout de quelques années, on s'aperçoit que tous les pays africains ne connaissent pas le même état d'avancement, ni les mêmes succès et/ou problèmes dans la mise en place et l'application effective du CDMT.

Qu'en est-il ainsi de son introduction et de sa mise en œuvre au Sénégal?

Pour réduire la pauvreté de moitié à l'horizon 2015, l'Etat sénégalais mettra en place de manière soutenue, une politique économique et sociale permettant de relever significativement ses performances socio-économiques et de placer le pays sur un sentier de développement humain durable ${ }^{12}$. Toutes ces raisons ont amené l'Etat Sénégalais à adopter de nouveaux outils de gestion budgétaire. C'est dans ce sens que le CDMT est introduit dans la gestion budgétaire. Conséquence du passage des budgets de moyens à ceux de résultats, le CDMT répond à l'exigence par l'UEMOA du respect du principe de bonne gouvernance et de transparence auquel tout Etat membre est soumis.

Houerou, R. Talierco, « Médium Term Expenditure Framework : preliminary lessons from Africa », Africa Region working paper, Wold Bank, fébriry, 2002.

8 Problème actuels des CDMT dans les pays africains, Rapport Atelier de la Banque mondiale, mai 2003, p.2.

9 Idem.

10 Une croissance plus forte, plus stable et plus réductrice de pauvreté, une approche participative, une gestion par résultat, un meilleur suivi.

11 Rapport Atelier de la Banque mondiale, Op. Cit. p.2.

12 Dans les réformes postérieures à la dévaluation, la pauvreté a fait l'objet d'une préoccupation centrale compte tenu de son ampleur et de son extension. Plusieurs actions ont été amorcées à travers la mise en œuvre de divers programmes spécifiques de lutte contre la pauvreté. En plus des programmes sectoriels sur la santé, l'éducation, les infrastructures de base etc., un plan de lutte contre la pauvreté (PLP) a été formulé en 1997. Cependant, les performances économiques enregistrées n'ont pas contribué à améliorer les conditions de vie des populations et à réduire substantiellement la pauvreté. Ce retour de la croissance enregistré sur la période 1995-2001 n'a pas suffit à garantir une réduction significative de la pauvreté. La faiblesse de l'investissement, l'atonie de l'agriculture et de l'industrie expliquent le contenu modeste en emploi de la croissance économique et sa faible propagation vers les populations les plus pauvres. cf. Document stratégique de réduction de la pauvreté (DSRP), SENEGAL, avril, 2002, p. 2. 
En effet, par CDMT, il faut entendre un outil de pilotage, un instrument stratégique de gestion des finances publiques. Un outil stratégique parce qu'il repose essentiellement sur la planification et la programmation économiques mais à moyen terme. A la différence des autres instruments de programmation comme la loi de programme qui est de 2 à 6 ans, le CDMT est un outil de planification à moyen terme (3 ans maximum). Cet instrument permet d'assurer une meilleure crédibilité du système budgétaire au niveau interne comme externe; d'améliorer la prévisibilité des ressources et des dépenses publiques, de renforcer la performance des gestionnaires des deniers publics à travers une discipline budgétaire plus rigoureuse, et enfin de donner les bases d'un suivi-évaluation axé sur les résultats à travers des indicateurs. Les innovations majeures dont cet instrument fait preuve, visent à modifier radicalement le mode précédent d'allocation et de programmation budgétaires bâti sur la mise à disposition de moyens.

La présente étude se propose d'analyser l'introduction des CDMT dans la gestion budgétaire sénégalaise dans le souci de comprendre le passage des budgets de moyens aux budgets de résultats (I), afin de pouvoir procéder à l'évaluation des questions concernant le problème de leur mise en œuvre (II).

\section{I // LES CDMT, UN OUTIL DE PERFORMANCE BUDGETAIRE AU SENEGAL.}

Les CDMT ont été introduits au Sénégal dans le souci, d'une part de mettre à la disposition du pays, un outil permettant de mieux gérer le déficit budgétaire à travers des projections réalistes concernant les recettes et, d'autre part, de faciliter l'identification des objets de dépenses prioritaires. Son adoption dans la gestion budgétaire sénégalaise qui repose essentiellement sur la recherche d'une intégration des politiques budgétaires (1) et sur des objectifs basés sur la performance (2), est consolidée par une dynamique normative nouvelle émergente dans le droit budgétaire sénégalais et communautaire (3).

1. Un outil d'intégration des politiques budgétaires sectorielles

Il y a eu, certes, au Sénégal, des tentatives d'élaboration de programmes sectoriels tels que dans les domaines de la santé, de l'éducation, etc., mais ces programmes restaient isolés et, par conséquent, ils ne tiennent pas compte des interactions intersectorielles.

La méthode CDMT pousse le processus programme sectoriel plus loin en ce qu'elle établit le lien entre les choix sectoriels et un cadre macroéconomique qui, fournit une indication quant à l'enveloppe des ressources. Par touches, les adaptations apportées aux dispositifs existant au Sénégal ${ }^{13}$ semblent inscrire les réformes entreprises dans un «processus de transformation du système financier public qui s'apparente à une sorte de transfiguration progressive de celui-ci $»^{14}$. Elles partent de l'idée «qu'un bon budget (...) est celui qui colle

13 BOUVIER (M) et alii, Finances publiques, Paris, LGDJ 5ème éd., 2000, p.23.

14 ORSONI (G), Science et législation financières, Paris, Economica, 2005, p.138. 
aux réalités du moment», c'est-à-dire qui intègre «les réponses suscitées par la conjoncture présente» ${ }^{15}$. En plus de ces soucis contingents, ces outils budgétaires prennent en compte le programme gouvernemental ${ }^{16}$, confirmant que «le budget est essentiellement un acte politique» ainsi que l'écrivait Gaston Jèze.

Au-delà de ces objectifs, une constante caractérise la doctrine budgétaire sénégalaise: l'assainissement. Ce qui justifie les plans d'action conçut par l'administration financière dans le but de renforcer la maîtrise des dépenses publiques, de même que la révision des procédures d'exécution des dépenses publiques. Ces initiatives ne peuvent se comprendre que par rapport aux faiblesses et aux dysfonctionnements constatés dans le système comptable antérieur, ainsi que dans le circuit des opérations budgétaires ${ }^{17}$ en usage jusqu'alors. Leur analyse fait ressortir que les lois de finances initiales n'assuraient plus effectivement leur fonction prévisionnelle, dans la mesure où le budget qu'elles contenaient, semblait avoir été conçu de manière fantaisiste, sans aucune chance de réalisation, sur des bases chiffrées non contrôlables. Les comptes publics n'étaient pas toujours fiables parce qu'ils ne présentaient pas la garantie de totalité qu'ils devraient avoir pour être crédibles. La transparence budgétaire ne se défendait plus, car l'information budgétaire manquait de clarté et de crédibilité. A l'égard du Parlement, cette situation était d'autant plus défavorable qu'elle conduisait à une dissimulation de la situation budgétaire réelle. L'autorisation budgétaire perdait alors tout son sens. Elle devenait «un simulacre». De ces constats, découle une interrogation fondamentale: comment maîtriser le budget?

La réponse apportée par les autorités gouvernementales sénégalaises, transparaît dans les doctrines budgétaires. A cet effet, la conception dégagée dès 2001, reste valable pour l'ensemble des lois de finances adoptées par la suite, car cette conception opérait une rupture fondamentale d'avec le passé et introduisait une nouvelle philosophie dans le processus d'assainissement des finances publiques. Cette conception, qui se traduit par un exercice de rigueur et de discipline et d'une gestion des ressources avec responsabilité, honnêteté, et efficacité, par l'ajustement des besoins aux moyens, conduit à une approche originale, à tout le moins singulière du processus budgétaire.

Au stade de l'élaboration, la méthode suivie se caractérise par une extrême prudence favorisant la prise en compte d'éléments susceptibles d'aider à concevoir un budget aussi proche que possible de la réalité. Au stade de l'exécution, des outils budgétaires et comptables sont mis en place pour maîtriser le circuit des opérations publiques, alors même que les procédures de contrôle fiscal sont renforcées.

La confrontation de cette optique avec les théories budgétaires, permet de constater que si le recours aux techniques classiques est indéniable, il est aussi fait appel à des méthodes

15 Gratuité de l'école, Décentralisation, Assurance maladie universelle, Projets d'infrastructures, etc.

16 FLINDE (A), « Rapport sur l'état d'avancement de la réforme sur le SIGFIP », in RFFP, n 98, 2007, p.48 cf. l'expérience sénégalaise du SIGFIP et de ASTER.

17 ARBOUSSET, « La violation de la sincérité budgétaire : un grief d'avenir? », RFFP, Nº74, Avril 2001, p.182. 
originales. En effet, les méthodes d'évaluation directe des ressources publiques constituent une mise en œuvre du principe de sincérité budgétaire, qui, demeure désormais le but poursuivi par les principes budgétaires traditionnels ${ }^{18}$. Innovation apportée par la loi organique 2011-15, l'introduction de ce principe de sincérité traduit une exigence de réalisme affirmé dans l'évaluation des ressources et des charges financières de l'Etat. Son article 30 énonce en effet que "les prévisions de ressources et de charges de l'Etat doivent être effectuées avec un réalisme et prudence, compte tenu des informations disponibles au moment où le projet de loi de finances est établi $\gg{ }^{19}$

Par contre, l'emploi des ressources prélevées, donne l'occasion d'opérer un véritable renouvellement des techniques. Le recours aux nouvelles technologies comme l'informatique vient appuyer le dispositif classique de la séparation des ordonnateurs et des comptables, pour obtenir une intégrité des comptes. Ce mécanisme complète le dispositif de régulation budgétaire et de gestion de la trésorerie qui astreint l'exécution du budget à une discipline.

En définitive, l'analyse de la doctrine budgétaire laisse transparaître une réforme ayant un objet global. Il est donc possible de soutenir que dans la quête d'une maîtrise des finances publiques, la doctrine budgétaire a conçu un budget rationalisé qui se dévoile a travers sa conception qui se veut sincère et son exécution, qui est enserrée dans une discipline rigoureuse. Ainsi donc, outre sa fonction intégratrice, le CDMT constitue un outil de pilotage budgétaire.

\section{Un outil de pilotage budgétaire}

Leur apparition récente vise d'une manière générale à contourner les contraintes découlant de la prévision budgétaire annuelle. Bâtis sur un horizon temporel de trois ans généralement et actualisé annuellement en fonction des réalisations ou des modifications des objectifs contenus dans le DSRP, les CDMT regroupent un ensemble cohérent, intégré et chiffré de programmes de dépenses publiques ventilés pour service dépensier et à travers lequel les Ministères opérationnels se conforment dans la prise de décisions de répartition et d'emploi des ressources. Ils poursuivent notamment l'objectif de l'emploi conforme et efficace des ressources par des services opérationnels qui jouissent d'une grande autonomie ${ }^{20}$. Les CDMT sont ainsi un dispositif de cadrage budgétaire contraignant. L'élaboration de cet outil représente une lourde tache sur le plan administratif et exige une planification rigoureuse et prudente de l'aide, une bonne appréciation des effets éventuels de la variation de la conjoncture internationale et une grande rigueur dans la détermination des choix des poli-

18 NOUPOYO (Gabriel), « Les nouvelles conditions de la politique budgétaire de sous-zone : étude des nouveaux instruments de rationalisation des choix budgétaire, le poids des conditionnalités externe », RFFP, $\mathrm{n}^{\circ} 98,2007, \mathrm{p} .88$.

19 Idem.

20 Ibid. 
tiques publiques de lutte contre la pauvreté, d'où l'importance de la phase de cadrage macroéconomique.

Par ailleurs, la banque mondiale encourage les pays africains de la zone franc à adopter du moins pendant une phase transitoire, une planification à moyen terme limitée aux dépenses sectorielles en charge de la lutte contre la pauvreté. L'objectif étant d'aboutir à terme, à des solutions débordant le cadre d'un seul secteur ${ }^{21}$. La consolidation du processus décisionnel dans le cadre de la préparation du budget annuel général, constitue ici une condition préalable. Les bailleurs de fonds préconisent une démarche altérative basée sur un système de programmation glissante. Ce mécanisme permet «une mise à jour constante du document stratégique par Pays, du volume des ressources et du programme indicatif selon les besoins et les résultats obtenus» $»^{22}$.

En dépit des difficultés inhérentes aux CDMT, pour reprendre M. NOUPOYO, ils constituent une référence pour la construction et la mise en œuvre des budget-programmes triennaux ${ }^{23}$. De manière générale, on observe un relatif consensus sur les objectifs théoriques et sur les explications «rationnelles» de l'introduction des CDMT au Sénégal. Le CDMT est un processus qui vise à faire évoluer un système budgétaire dans une triple direction $^{24}$ :

- définir un cadre pluriannuel de ressources stable, cohérent et réaliste pour éviter le plus possible que les impératifs de court terme dominent les décisions budgétaires, que des coupures soudaines de crédits créent des incertitudes et des comportements négatifs, que les fonds destinés aux service d'exécution et aux collectivités locales arrivent de façon tardive et irrégulière;

- favoriser une approche par programme avec des stratégies et des objectifs de performances clairement affichés;

- améliorer l'information en suscitant une mesure de coûts et des résultats et éclairant ainsi le système de décision et de gestion.

Nous constatons ainsi, que les enjeux majeurs de l'adoption des CDMT au Sénégal consistent à garantir durablement des allocations budgétaires conformes aux priorités stratégiques et à favoriser un emploi des ressources plus efficace et plus efficient par des agences d'exécution jouissant d'une plus grande autonomie et responsabilisation pour atteindre des résultats aux meilleurs coûts. Cette nouvelle dynamique budgétaire implique une mise en place d'un cadre juridique approprié, chose à laquelle et sous l'impulsion de l'UEMOA, le Sénégal n'a pas manqué.

21 Douze Ministères sont sous CDMT au Sénégal, cf. Evaluation des CDMT au Sénégal, Rapport de Séminaire de Saly, janvier 2010.

22 C'est la méthode appliquée au Sénégal.

23 MOREAU (F), «Implications des acteurs non- étatiques dans le processus de programmation : une évaluation préalable ", Le Courrier ACP-UE, n 199, juillet- aout 2003, p. 18.

24 NOUPOYO (Gabriel), Op. Cit, p. 89. 
3. Un outil exigeant une nouvelle dynamique normative

Cette exigence s'opère d'abord au niveau communautaire par des réformes du cadre juridique budgétaire. En effet, l'UEMOA avait apporté un cadre juridique destiné à garantir une meilleur transparence des politiques budgétaires, donc d'en permettre un contrôle effectif grâce à la comparabilité des documents élaborés. Toutefois, toutes les réformes introduites sont restée dans le cadre d'un budget de moyen, en outre elles n'ont pas totalement remédié aux faiblesses des différents systèmes budgétaires, caractérisés encore par une démultiplication du circuit de financement ${ }^{25}$. A cet égard, M. DUPRAT souligne que «les exigences concernant les ressources allouées se trouvent à l'origine d'une plus grande complexité du dispositif budgétaire, avec l'introduction du mécanisme des budget-programmes, induisant la logique de résultat. Il ne faut donc pas s'étonner de l'existence d'ajustements successif pour améliorer les solutions adoptées ».

L'UEMOA est engagée ainsi, depuis quelques années, dans un vaste chantier d'harmonisation des règles en matière de finances publiques pour ses huit Etats membres. Dans ces pays qui paraissent valoriser l'approche juridique, en adoptant des textes sur lesquels appuyer l'innovation budgétaire, la dimension expérimentale se trouve fortement présente. Tel fut le cas de la construction des programmes et de leur articulation avec les missions, de même que l'édification des budgets opérationnels de programme. Si pour les Etats africains, la dimension expérimentale semble prévaloir, il ne faut pas oublier que l'innovation découle alors d'accords internationaux, voire d'un mécanisme intégré donnant lieu à l'élaboration de directives à la destination des Etats, ainsi que pour l'UEMOA de la directive de 1999, modifiant celle de 1997, relative aux lois de fiances. Elle a adopté également la directive $\mathrm{N}^{\circ} 06 / 2009 / \mathrm{CM} / \mathrm{UEMOA}$, portant loi de finances au sein de l'UEMOA dont l'article 12 définit les programmes en relation avec les missions. En effet, à partir de son alinéa 2, l'article énonce que «(...) A l'intérieur des Ministères, les crédits sont décomposés en programmes, (...). Un programme regroupe les crédits destinés à mettre en ouvre une action ou un ensemble cohérent d'action représentatif d'une politique clairement définie dans une perspective de moyen terme. A ces programmes, sont associés des objectifs précis, arrêtés en fonction de finalité d'intérêt général et des résultats attendus. Ces résultats, mesurés notamment par les indicateurs de performance élaborée enfin de gestion par les ministères et institutions constitutionnelles concernées (...) » ${ }^{26}$.

Cependant, la délimitation claire des programmes se heurte également à la notion de programmes dits «fonction support», c'est selon une politique publique prédéfinie. Il s'agit des fonctions transversales, généralement appelées dotation dans la zone UEMOA. Cette pratique contredit la finalité des programmes de décrire des parties de politique publiques,

\section{Rapport Atelier de la Banque mondiale, Op. Cit, p.2.}

26 BOUVIER (M), La gestion de la dépense publique dans les pays de l'Afrique francophone subsaharienne, cité par DUPRAT (Jean Pierre), « La dynamique des réformes budgétaires : globalisation des problèmes, unification des outils et adaptation nationale des solutions ", RFFP, ${ }^{\circ} 98$, juin 2007, p. 19. 
conformément à l'article 14 de la directive $\mathrm{N}^{\circ} 06-2009$ qui précise que «les crédits budgétaires non répartis en programme sont répartis en dotations. Chaque dotation regroupe un ensemble de crédits globalisés destinés à couvrir des dépenses spécifiques auxquelles ne peuvent être directement associés des objectifs de politiques publiques ou des critères de performance(...) ».

Ce cadre normatif communautaire est transposé dans le droit budgétaire sénégalais par des lois organiques. Ainsi, la loi n²011-15 du 8 juillet 2011 relative aux lois de finances vise à transposer dans notre droit interne la directive précitée et abroge, par conséquent, la loi organique $n^{\circ} 2001-09$ du 15 octobre 2001 relative aux lois de finances, modifiée par la loi organique $\mathrm{n}^{\circ}$ 2007-29 du 10 décembre 2007. Ce dispositif juridique, renforcé par toute une série de textes de nature diverse, témoigne avec force de l'adhésion du Sénégal à la nouvelle approche de la gestion budgétaire orientée par les résultats. Ainsi nous pouvons dire que, enfin de compte, le renouveau de ce cadre juridique répond au souci d'adapter l'allocation des ressources publiques à la réalisation des objectifs de développement du Sénégal. Néanmoins, la mise en œuvre de ces outils de performance budgétaire appelle un certain nombre de défis à relever par l'Etat du Sénégal.

\section{II // LES DEFIS REELS DE L'APPLICATION DES CDMT AU SENEGAL}

Le Sénégal est confronté, dans la mise en œuvre des CDMT à l'expérimentation de nouvelles techniques d'élaboration budgétaire (1), à des contraintes à la fois d'ordre technique et structurel (2). Ainsi, le Sénégal doit généralement faire des efforts dans deux sens : le cadrage macroéconomique et la programmation sectorielle (3).

\section{L'expérimentation de nouvelles techniques d'élaboration budgétaire}

Actuellement ${ }^{27}$, douze ministères du gouvernement sénégalais sont sous CDMT. Normalement, le CDMT est un processus qui comprend plusieurs étapes et qui agit sur tout le cycle budgétaire: il modifie complètement la phase de préparation et influe significativement sur la phase d'exécution et suivi- évaluation ${ }^{28}$. L'élaboration d'un CDMT est processus complexe qui comprend généralement les étapes suivantes ${ }^{29}$ :

- Prévision de ressources mobilisable et plafonnement sectoriel préliminaires;

- Elaboration des CDS-MT préliminaire;

- Débats et révision des plafonds budgétaires sectoriels;

- Approbation des plafonds sectoriels par Ministères par le conseil des Ministres

- Finalisation des CDS-MT;

- Finalisation du CDMT et approbation par le Conseil des Ministères et le Parlement.

27 Janvier 2010.

28 DUPRAT (Jean Pierre), « La dynamique des réformes budgétaires : globalisation des problèmes, unification des outils et adaptation nationale des solutions », Op. Cit., p.15.

29 Idem. 
L'élaboration de cet outil présente une lourde tâche sur le plan administratif et exige une planification rigoureuse et prudente de l'aide, une bonne appréciation des effets éventuels de la variation de la conjoncture internationale et une grande rigueur dans la détermination des choix de politiques publiques de lutte contre la pauvreté. D'où l'importance de la phase de cadrage macro- économique ${ }^{30}$. Ce cadrage macro- économique suppose la définition des missions dudit secteur ainsi que la détermination des acteurs externes et de leur contribution dans la réalisation de ces missions. Il faut comprendre par mission en effet, le but général poursuivi par l'entité. Il s'agit de présenter la vision à plus ou moins long terme. C'est la valeur ajoutée du secteur à la réalisation des objectifs de développement du gouvernement $^{31}$. De ce fait, le processus de ventilation des ressources budgétaires commence par la définition, par le Ministère des Finances, du cadre macroéconomique. A l'origine, il était envisagé de recourir à un cadre macroéconomique pour estimer l'enveloppe totale des ressources en tenant compte de variables pertinentes telles que la croissance du PIB, les taux d'inflation, la croissance de la masse monétaire, etc. ${ }^{32}$ En outre, une analyse des résultats du secteur s'impose. Elle permet de retracer l'évolution de l'organisation, des réformes mises en œuvre, de faire le bilan de la politique de financement etc., d'allocation des ressources, de prendre en compte les leçons tirées de la Revue des Dépenses Publiques; de déterminer les programmes et projets exécutés en cours, ayant contribué à la réalisation des résultats globaux atteint par le secteur en cours de la période passée en revue ${ }^{33}$. Toujours, dans cette même lancée de pré-élaboration du CDMT, il convient de souligner les principales contraintes et les défis auxquels le secteur reste confronté malgré les programmes qui ont été mise en œuvre dans le passé et l'intervention des autres acteurs impliqués dans la réalisation de la mission de l'entité auquel s'applique le CDMT ${ }^{34}$.

Il faut aussi rappeler les objectifs et indicateurs de résultats du secteur d'où les objectifs sectoriels sont présentés en détails dans les documents de programmes sectoriels et sont repris en général dans le DSRP. Leur définition relève de la planification stratégique et ces objectifs doivent garder toute leur articulation et cohérence avec les missions assignés au secteur. Les indicateurs de résultats quant à eux, permettent de mesurer en terme quantitatif ou qualitatif les changements apportés dans le secteur à travers la mise en œuvre de programmes et de projets jugés prioritaire ${ }^{35}$. Sont également déterminés, les programmes et projets à réaliser sur la période à la suite des arbitrages internes (avant les conférences bud-

30 Rapport Atelier de la Banque mondiale, Op. Cit, p. 12.

31 Concepts et Démarche d'élaboration du CDMT, Séminaire du 20 octobre au 6 novembre 2009, Saly Portudal, Sénégal.

32 NOUPOYO (Gabriel), Op. Cit. p.88.

33 Voire, Document sur l'application de la Gestion axée sur le résultat au domaine budgétaire, SENEGAL, 23 février 2010, p. 26.

34 ARMAH (Bartholomew), Le cadre des dépenses à moyen terme : cas du Ghana, Institut of Economica Affair, p.3.

35 Document sur l'application de la Gestion axée sur le résultat au domaine budgétaire, Op. Cit. p. 27. 
gétaires), des ajustements opérés après que le plafond budgétaire soit notifié au Ministère concerné par le secteur. Ainsi donc la méthode CDMT fixe les plafonds pour les dépenses des DSM pour la période triennale comprend l'ensemble des dépenses. A la suite, un tableau récapitulatif donne les indicateurs sur l'intitulé des programmes-projets, des objectifs sectoriels auxquels ils contribuent, des objectifs spécifiques propres, des composantes, des services responsables, des extrants attendus et des allocations budgétaires sur la période du CDS-MT ${ }^{36}$.

Enfin, des mécanismes de mise en œuvre et de suivi-évaluation sont prévus. Normalement, le CDMT est un processus qui comprend plusieurs étapes et qui agit sur tout le cycle budgétaire. Il apparait comme un instrument de pilotage qui ne supprime pas le cycle budgétaire annuel, mais le renforce et lui donne de crédibilité et d'efficacité.

\section{Une difficile appropriation de l'outil CDMT}

La déficience des outils indispensables à la programmation financière, constitue une difficulté dans la mise en œuvre des CDMT. Il est certain que la qualité des indicateurs nous renseigne sur l'ambition des objectifs et la crédibilité des politiques publiques, sur la capacité des Etats à cibler la pauvreté et enfin sur le niveau d'efficience de la dépense publique $^{37}$. Or, la construction des budgets-programmes est largement obérée par la difficulté dans la collecte d'informations nécessaires à l'élaboration des indicateurs de lutte contre la pauvreté ${ }^{38}$. L'absence de base de donnés identifiées ne facilite pas non plus la maîtrise des coûts de " production » de certains services publics (santé, éducation). L'inadaptation de certains indicateurs aux objectifs des programmes, confirme la déconnexion des programmes des objectifs poursuivis, et contribue ainsi à amplifier le sentiment d'une gestion strictement technicienne de la réforme ${ }^{39}$.

En effet, l'exactitude dans l'estimation de coûts de revient des politiques et des programmes est également indispensable pour mieux définir les priorités budgétaires. Seulement, les difficultés d'établissement et de prévision d'évolution des valeurs d'indicateurs proviennent quelquefois de l'inertie et l'incapacité de certains administrations à identifier et à présenter des projets ou programmes et dans la plupart des cas du sous-équipement.

Par ailleurs, à la suite de ces entraves structurelles, s'ajoutent celles d'ordre technique, relatives à la difficile insertion de ces instruments programmation. De ce fait, la nouvelle architecture, conçue à l'intention de l'Etat en vue de préserver la discipline budgétaire et d'assurer la réalisation cohérente des mesures contenues dans les DSRP, souffre de nombreux dysfonctionnement qui sapent la portée du dispositif. Ces atteintes se traduisent en

36 Idem, p.28.

37 Ibid., p.29.

38 Ibid., p.31.

39 BALMES (J-C), «Le point sur ... les indicateurs de suivi des politiques d'universalisation de la scolarisation primaire ", La Lettre des économistes de l'AFD, n 2, 2003, p. 5. 
pratique par un décalage entre les dotations financières inscrites dans les CDMT et celles prévues par la loi de finances. Elles se traduisent également par une mauvaise articulation des instruments de programmation à moyen terme des dépenses publiques, par le dépassement des ministères sectoriels des plafonds de dépenses inscrits au sein des CDMT. Ces défaillances découlent d'une part, de l'absence d'un support juridique pour assurer le pilotage des réformes, d'autre part, par le non synchronisation du calendrier budgétaire et enfin de la centralisation du processus d'élaboration de budget ${ }^{40}$.

Au niveau des structures administratives, face à la pénurie de ressources humaines, le renforcement du processus décisionnel dans la phase de préparation et d'élaboration du budget s'impose pourtant. La tendance actuelle semble plutôt s'orienter vers une réorganisation institutionnelle en vue de parvenir à l'unification effective des procédures d'exécution du budget.

Par ailleurs, la mutation de la mission du plan vers des compétences de réflexion stratégique bouleversera le rôle et l'organisation des structures politiques qui en auront la charge. C'est dans ce contexte qu'on assiste déjà au recentrage des missions du Ministère du plan au Sénégal, désormais chargés de définir les stratégies globales sur le plan national, d'aider les cellules de programmation des ministères sectoriels à élaborer leurs stratégies sectorielles et leurs actions prioritaires à travers la production statistique, d'assurer la coordination de la programmation des investissements publics, la recherche des ressources de financement et le suivi-évaluation de la mise en œuvre des programmes de développement ${ }^{41}$.

\section{$3^{\circ}$ ) Pour un lendemain meilleur des CDMT au Sénégal}

A l'intérieur d'un système développé de gestion par objectifs se produit une quantité d'informations sur les résultats et des coûts qu'il faut analyser au niveau ministériel, au service des décideurs politiques. En l'absence d'une telle analyse, «un bouclage» du système de flux d'informations et de directives politiques, le système de gestion tout entier risque de rester un exercice technique sans rapport avec le processus politique.

Pour exploiter les possibilités de la gestion par objectifs, un certain nombre d'analystes doivent assister la direction politique dans le rôle de «traducteurs» entre le niveau politique et le niveau administratif. Ces exigences posent le débat de la nécessité des éléments techniques fondamentaux à l'efficacité des CDMT. En outre, le CDMT doit conduire les responsables sénégalais à améliorer l'analyse et la gestion sur de nombreux points et même dans certains cas à modifier significativement le système de planification-budgétisation du pays ${ }^{42}$.

40 NOUPOYO (Gabriel), Op. Cit. p.93.

41 REVERCHON (A), « La réforme de l'Etat risque de déraper », Le Monde " Economie, 24 Février 2004, p.8.

42 BATOUM- BA- NGOUE (S. T), « Démocratie et processus budgétaire dans les Etats de la communauté économique et monétaire de l'Afrique centrale : le cas du Cameroun ", AFRILEX, n 4 , pp. 12 et ss. Cité par NOUPOYO (Gabriel), Op. Cit. p. 95. 
Ainsi, à l'intard des pays qui ont mis en place des CDMT pour fixer des enveloppes sectorielles à moyen terme, le Sénégal doit généralement fait des efforts dans deux sens : le cadrage macroéconomique et la programmation sectorielle. En terme de cadrage macroéconomique, comme la plupart des pays, les autorités sénégalaises tentent d'appliquer des modèles quasi-comptables sur la base de projections financières de type FMI ou des modèles de type Banque Mondiale ${ }^{43}$. La réalité de nombreux pays est que les modèles macroéconomiques existants ne sont pas opérationnels, soit qu'ils ne sont plus adaptés, soit que la base d'informations est défaillante, soit que le projet d'appui et /ou les cadres formés ne sont plus là. Généralement, l'enveloppe des ressources est basée sur les projections de croissance du FMI, ce qui nuit à la crédibilité du CDMT, car elle n'est pas toujours garante de réalisme et conduit à une interprétation très étroite du cadre macroéconomique et souvent à des distorsions avec la philosophie et les objectifs des DSRP.

En terme, de programmation sectorielle, tous les pays qui ont élaboré des CDMT sectoriels ont introduit une méthode de planification stratégique qui inclut le choix des stratégies et une analyse des coûts. Il se pose alors la question de savoir comment prioriser les actions et choisir les dépenses publiques sectorielles? Il y a un certain nombre d'éléments préalables à prendre en compte, à mener qui ne doivent pas être sous estimées et qui peuvent se résumer comme suit :

- un bilan détaillé qui comprend l'analyse des politiques et dépenses publiques, la justification des interventions publiques, l'évaluation des rôles (public, privé, non lucratif, privé lucratif) les plus efficaces.

- un cadre de réflexion multidimensionnel qui permet de s'assurer que toutes les dimensions (financières, économiques, intentionnelles, sociales, culturelle, etc.) et que des questions multisectorielles sont prises en compte dans tous les CDMT sectoriels.

- une analyse multicritères des dépenses publiques sectorielles selon une méthodologie normalisée pour tous les secteurs.

Au-delà de cette approche de performance, il convient d'insister, pour que la déclinaison de la performance soit opérationnelle, sur la nécessité de sectionner des objectifs reflétant les priorités de chaque programme de politique publique et découlant préalablement de l'analyse stratégique du programme. Il importe d'éviter une tendance naturelle à une inflation relative au nombre d'objectifs et d'indicateurs. En revanche, retenir un nombre limité d'objectif dont le libellé est clair et compréhensible par l'ensemble des acteurs chargés de mettre en œuvre telle ou telle politique publique est l'un des facteurs de réussite de la mise en œuvre d'une démarche de performance dans les administrations publiques sénégalaises. De même les précisions à apporter sur les leviers d'actions nécessaires à l'atteinte des objectifs ainsi que les raisons du choix des indicateurs contribuent à la réussite de la démarche.

43 Modèle de la Banque mondiale : Revised Minimum Standard Modèle (RMSM) ou Modèle conjoint Banque mondiale- FMI : Revised Minimum Standard Modèle eXtended plus Poverty $(\mathrm{RMSM}-\mathrm{X}+\mathrm{P})$. 
Mesurer la performance est donc devenue une exigence pour la gestion publique, non seulement pour des raisons de contraintes des finances publiques, mais également pour maintenir des services publics de qualité et si possible à moindre coûts. De façon plus générale, c'est une exigence démocratique impliquant un changement de culture et une modification sensible des comportements.

\section{CONCLUSION}

La démarche sénégalaise de performance dans le cadre des finances publiques, que d'autres pays ont déjà engagée, est conçue non seulement comme devant conduire à une meilleure allocation des moyens budgétaires mais également comme instituant un dispositif de pilotage ayant pour but d'améliorer l'efficacité de la dépense publique en orientant la gestion publique vers des résultats prédéfinis. La méthode CDMT pousse le processus programme sectoriel plus loin en ce qu'elle établit le lien entre les choix sectoriels et un cadre macroéconomique qui, fournit une indication quant à l'ensemble de l'enveloppe des ressources. Ce faisant, le concept déjà ancien "d'Etat- stratège », comme le souligne Jean- Pierre DUPRAT, se trouve placé au premier plan, entrainant une remise en cause du cadre administratif traditionnel.

Toutefois, le Sénégal est confronté, dans la mise en œuvre des CDMT à des contraintes à la fois d'ordre technique, structurelle et humain. Ces atteintes se traduisent en pratique par un décalage entre les dotations financières inscrites dans les CDMT et celles prévues par la loi de finances. Elles se traduisent également par une mauvaise articulation des instruments de programmation à moyen terme des dépenses publiques, par le dépassement par les ministères sectoriels des plafonds de dépenses inscrits au sein des CDMT.

Pour exploiter les possibilités de la gestion axée sur le résultat, un certain nombre d'analystes doivent assister la direction politique dans le rôle de «traducteurs» entre le niveau politique et le niveau administratif. Ces exigences posent le débat de la nécessité des éléments techniques fondamentaux à l'efficacité des CDMT sectorielles à moyen terme. A l'intard des pays qui ont mis en place des CDMT pour fixer des enveloppes sectorielles à moyen terme, le Sénégal doit généralement faire des efforts dans deux sens : le cadrage macroéconomique et la programmation sectorielle. 